\title{
weil dies ist ^MElnungsbezogen Über die verfestigte Verbzweitstellung in meinungs- und gefühlsbezogenen weil-Sätzen im gesprochenen Deutsch
}

\author{
Dario MARIĆ
}

\begin{abstract}
Weil dies ist ${ }^{\wedge}$ MEInungsbezogen. On the solidified verb in the second position in the weil-sentences in German spoken language

This paper explores utterances in the form of causal clauses introduced by the conjunction weil in conversational German. The focus of this research is the relationship between the position of the finite verb in the clause and the type of explanation or clarification uttered in authentic conversation. The research shows that in addition to a previously recognized category of weil-utterances with a finite verb in the second position in the clause, there is also a category of weil-utterances of this type that express personal opinion or personal feeling.
\end{abstract}

Keywords: weil-utterances, German spoken language, verb in the second clause position, verb in the final clause position, meaning- and feeling-related utterances

DOI: doi.org/10.15452/StudiaGermanistica.2020.27.0002

\section{Einleitung}

Die Subjunktionen weil, obwohl, wobei verbinden sowohl im geschriebenen als auch im gesprochenen Deutsch den Inhalt eines untergeordneten ${ }^{1}$ Teilsatzes mit Verbletztstellung mit dem Inhalt eines ihm übergeordneten, unmittelbar vorausgehenden oder unmittelbar folgenden Teilsatzes. Ihre Homonyme wiederum, die Diskursmarker weil, obwohl, wobei knüpfen Äußerungen mit Hauptsatzwortstellung an das davor Geäußerte an (z. B. in Was wär' denn deiner Meinung nach nicht okay? Weil du hast ja vorhin gesagt, er nervt dich ganz schön. aus Günthner 1996:327).

In den grundlegenden Untersuchungen der weil-Sätze im gesprochenen Deutsch wurden verschiedene Arten von Begründungen bzw. Erklärungen als Gründe für Unterschiede in der Wortstellung angenommen (Günthner 1993 und 1996). In der weiteren Forschung wurden diese Stellungsverhältnisse und der Grad der prosodischen Integration solcher weil-Sätze in den vorhergehenden Teil der Äußerung genereller mit dem Funktionspotential dieser grammatischen Mittel in Beziehung gesetzt (Scheutz 1998 und 2001). Dadurch lockerte sich die Auffassung von einem engen

Für ihre Verbesserungsvorschläge bedanke ich mich bei Prof. Dr. Manuela Caterina Moroni und bei den beiden Rezensenten. 
Verhältnis zwischen dem Begründungstyp und der Verbstellung des weil-Satzes. Da sich dabei zeigt, dass die Äußerungen mit beiden Wortstellungstypen und bei unterschiedlicher prosodischer Integration den gleichen Begründungstyp ausdrücken können, bleibt unklar, durch welche anderen Faktoren die Wahl der Wortstellung bedingt sein könnte. (Siehe propositionales weil bei Verbletztund Verbzweit-Sätzen in Scheutz 1998:95-99 und 101-105).

In dem vorliegenden Beitrag wird angenommen, dass die Verbzweitstellung in weil-Sätzen zum Teil ein Zeichen dafür ist, dass es sich um Begründungen handelt, die nicht vom Beginn des (behauptenden) Vor-Kontextes an vorgesehen waren, sondern deren Einfügung an dieser Stelle im Verlauf ad hoc realisiert wird. Zum anderen wird auf Verwendungen mit verfestigten Stellungsmustern hingewiesen, mit denen Begründungstypen zum Ausdruck gebracht werden, die zum Teil in der Forschung bisher nicht erfasst wurden. Letztlich wird dafür plädiert, das sogenannte konversationelle Fortsetzungssignal weil (Gohl/Günthner 1999:51-53, oder „Diskurspartikel“ bei Scheutz 1998:107-108), dem keine Begründung oder Erklärung folgt, als den Anfang eines geplanten, dann aber abgebrochenen Äußerungsteils mit diesem Konnektor zu verstehen. Die Analysen in diesem Beitrag beziehen sich auf ein Korpus von hundert weil-Äußerungen aus Gesprächen im Rahmen der Phone-in-Sendung ,Im Gespräch des Deutschlandradios.

\section{Ziele}

Zentrales Ziel der vorliegenden Arbeit ist eine Übersicht über die Funktionen der weil-Äußerungen im gesprochenen Deutsch in ihren verschiedenen Verwendungstypen wahrzunehmen. Damit verbunden ist die Intention, hier funktionale Kategorien in einer Weise darzustellen, dass sie sich zur didaktischen Aufbereitung im DaF-Unterricht eignen. Das ist gerade in diesem Fall bedeutsam, da nach wie vor gerade Ergebnisse des Gesprochene-Sprache-Forschung wenig Eingang in Unterrichtsmaterialien finden. Gerade sie könnten aber den DaF-Lernenden Möglichkeiten der Formulierung aufzeigen, von denen die mündliche Kommunikation erheblich profitieren könnte. In diesen Kontext gehört auch, dass die funktionalen Kategorien möglichst in einer Weise benannt werden sollten, die auch ohne vertiefte linguistische Ausbildung verstanden und memoriert werden können.

\section{Analyseergebnisse}

Für die bekannten Arten der Begründungen wie für Rechtfertigung der eigenen, unmittelbar davor geäußerten Sprechhandlung (sog. sprechhandlungsbezogene Kausalrelation, für ein Beispiel siehe weiter unten Transkript 1) oder für die Stützung eigener, davor geäußerten „Behauptung bzw. Vermutung durch die Angabe von Voraussetzungen/Evidenzen/Annahmen“ (Scheutz 1998:105) (sog. epistemische Kausalrelation, für ein Beispiel siehe weiter unten Transkript 2) oder für eingeschobene, für das Verständnis der Erzählung notwendige Zusatzinformation (für ein Beispiel siehe weiter unten Transkript 3), die durch weil-Äußerungen mit Verbzweitstellung ausgedrückt werden, konnten im Korpus dieser Studie wiederholt Belege gefunden werden. Neben diesen bildete sich im Korpus dieser Studie eine Gruppe der weil-Äußerungen mit Verbzweitstellung heraus, die subjektive Meinungsäußerungen oder deren Teile und Gefühlsausdrücke darstellen. Diese weil-Äußerungen begründen die Proposition des Vorgängersatzes. Ihnen gehen keine Denkprozesse symptomatisierender Verzögerungssignale unmittelbar voraus und sie weisen selbst keine solchen Verzögerungssignale oder Pausen nach weil auf (für den Intonationsverlauf einer solchen weil-Äußerung siehe Abb. 1.). Für die Gruppe der weil-Äußerungen mit Verbzweitstellung aus dem Korpus, denen Verzögerungssignale unmittelbar vorausgehen oder die selbst Verzögerungssignale oder Pausen nach weil enthalten, wird angenommen, dass ihr Unterbrochensein und ihre Verbstellung durch einen relativ späten Planungsmoment bedingt sind (für den Intonationsverlauf einer solchen weil-Äußerung siehe Abb. 2.).

Die nächsten Unterpunkte dieses Kapitels bringen jeweils die Beispiele für die Gruppen der weil-Äußerungen mit Verbzweitstellung aus dem Korpus und ihre kurze Beschreibung, und zwar zunächst die bisher in der Fachliteratur erfassten Gruppen und anschließend die bisher nicht erfassten. 


\subsection{Die bisher erfassten Begründungstypen in Form von weil-Sätzen mit Verbzweitstellung}

Das folgende Transkript 1 stellt einen Ausschnitt aus einem Gespräch über die Gültigkeit eines Behindertenparkausweises außerhalb des eigenen Bundeslandes dar (für die Transkription der Gespräche aus dem Korpus wurde GAT 2 von Selting et al. 2009 benutzt). Durch die weil-Äußerung mit Verbzweitstellung in Zeile 19 wird eine sprechhandlungsbezogene Kausalrelation realisiert. Konkret wird durch diesen weil-Satz ein eigener, unmittelbar davor geäußerter Ratschlag an den Anrufer, sich nochmals genauer zu erkundigen, in Bezug auf eigene Vorerfahrungen mit verschiedenen deutschen Bundesländern, begründet. Im vorhergehenden Teil des Gesprächsausschnitts hatte sich der Anrufer darüber beklagt, dass es seinem Schwager unmöglich sei, seinen Behindertenparkausweis in manchen deutschen Bundesländern (Rheinland-Pfalz und Berlin) zu benutzen.

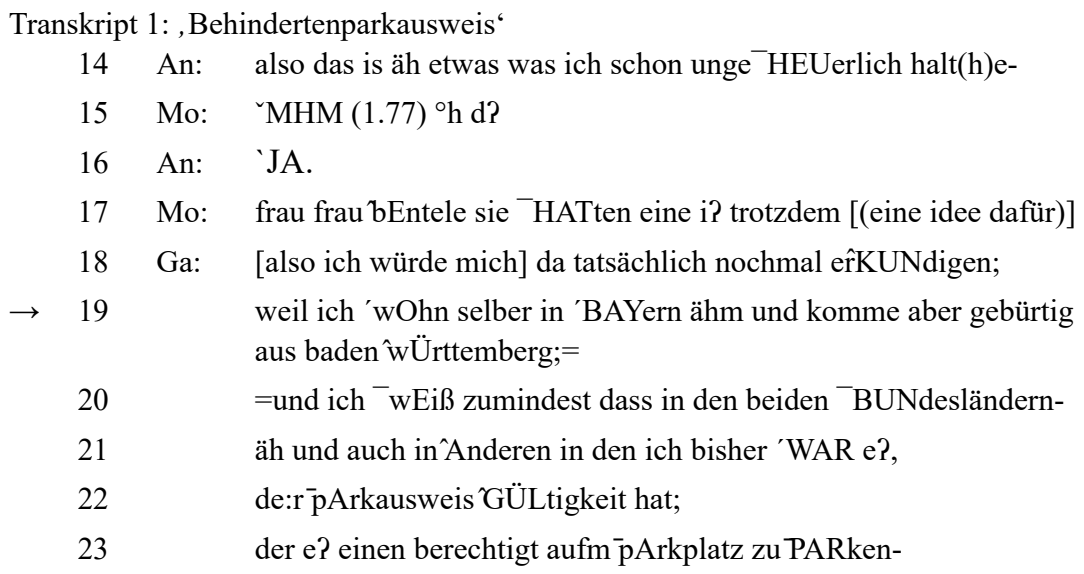

Der Gast im Studio rät dem Anrufer in Zeile 18 sich zu erkundigen, ob Behindertenparkausweise tatsächlich nur für das Bundesland gelten, in dem sie ausgestellt wurden, und drückt gleichzeitig eigene Zweifel an einer solchen beschränkten Gültigkeit der Behindertenparkausweise aus. Die Behauptung, zu wissen, dass in Bundesländern, in welchen der Gast der Sendung war, die Behindertenparkausweise aus anderen deutschen Bundesländern gelten (Z. 19-23), liefert eine Berechtigung für den Ratschlag, sich nochmals zu erkundigen.

Ein Beispiel für die Verwendung eines weil-Satzes mit epistemischem Kausalverhältnis zur Vorgängeräußerung beinhaltet das folgende Transkript (Z. 14) aus einem Gespräch zwischen der Anruferin und dem Pflanzenarzt über das durch Würmer befallene Fleißige Lieschen.

Transkript 2: , der Wurm

$12 \mathrm{Ga}$ : en? en? man müsste den diesen wurm dann mal SEhen un? und mal gucken wie grOß un_was is_es ob das vielleicht auch äh ja eher ne $\uparrow$ '1Arve ist die sich $\uparrow{ }^{\prime} w E$ Eiterentwickelt und die die richtig frisst also; $h$

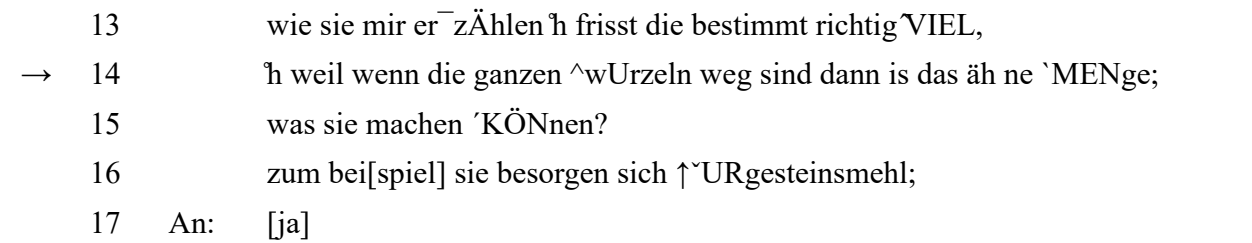

Im Vorgespräch des Gesprächsteils aus dem Transkript 2 berichtet die Anruferin von ihrem Problem mit durch Würmer angefressenen Wurzeln ihrer Fleißigen Lieschen auf der Fensterbank. Die Feststellung des Pflanzenarztes aus der Zeile 13, dass diese Würmer viel fressen, begründet er, indem er in Zeile 14 die Beobachtung der Anruferin, dass die ganzen Wurzeln ihres Fleißigen Lieschens aufgefressen sind, wiederholt, bevor er in Zeile 15 einen Rat ankündigt und ihn in Zeile 16 auch gibt. 
Im Ausschnitt aus einem Gespräch über Erfahrungen bei der Leitung eines deutschen Filmfestivals teilt dessen Direktor seine Erfahrungen mit Regisseuren, Filmproduzenten und Schauspielern mit, die nicht selten durch Erpressungen und kurzfristige Absagen der prominenten Gäste und Teilnehmer gekennzeichnet seien.

Transkript 3: ,Filmstars`

06 Ga: da wird šhr viel ge'DROHT,

07 ich ziehe meinen ${ }^{-}$fllm zu'RÜCK;

08 die 'SCHAUspielerin kommt nich;

09 der ,creaky>STAR> kommt nich;

$10 \quad{ }^{\circ} \mathrm{hh}$ und sie sitzen natürlich 'dA ${ }^{\circ} \mathrm{hh}$ und sind !HILFlos!.

$\rightarrow \quad 11 \quad$ weil wir be $\uparrow^{\uparrow} Z$ ZAHlen ja diese leute nicht.

12 das bezahlen ja die $\uparrow^{\wedge} A N d e r e n$.

In Zeile 10 stellt er die Hilflosigkeit der Organisatoren bei Absagen der Gäste und Teilnehmer des Filmfestivals fest. In der weil-Äußerung mit Verbzweitstellung in Zeile 11 wird eine für das Verständnis notwendige Zusatzinformation gegeben, und zwar der Umstand, dass diese Personen nicht von den Festivalveranstaltern für das Erscheinen auf dem Festival bezahlt werden. Diese Zusatzinformation erklärt, warum sie im Nachhinein ein solches Benehmen nicht sanktionieren lassen können und dadurch in Zukunft verhindern können und ist nicht der direkte Grund für die Hilflosigkeit der Festivalveranstalter.

Vereinzelt kommen im Korpus auch weil-Äußerungen mit Verbletztstellung vor, die wie die in den bisherigen Fällen angeführten weil-Äußerungen mit Verbzweitstellung eine sprechhandlungsbezogene, eine epistemische Kausalrelation oder eine für das Verständnis notwendige Zusatzinformation ausdrücken können.

\subsection{Meinungs- bzw. gefühlsbezogene weil-Sätze mit Verbzweitstellung}

Mit der weil-Äußerung in Zeile 14 des folgenden Transkripts 4 aus einem Gespräch über die nichtchemische Bekämpfung von Schädlingen bei der Pflanzenzucht begründet ein Gärtnermeister sein in Zeile 13 formuliertes Plädoyer für dieses Vorgehen. Zu diesem Zweck führt er eine aus seiner Sicht wichtige Voraussetzung für eine - wenn auch etwas weniger wirksame - nichtchemische Behandlung von Pflanzen auf, nämlich die Fähigkeit der Pflanzen, mit einer gewissen Menge von Schädlingen zu leben. Es handelt sich bei der Begründung um die Äußerung seiner eigenen (für mich) Meinung über die Wichtigkeit der genannten Fähigkeit der Pflanzen. In Zeile 15 kommt ein dass-Satz mit Verbzweitstellung vor (siehe dazu Freywald 2009).

Transkript 4: ,Zusammenleben mit den Schädlingen`

$13 \mathrm{Ga}$ : man sollte halt versUchen wirklich ${ }^{\circ} \mathrm{h}$ mit mit na个`TÜRlichen

mitteln ${ }^{\circ} \mathrm{h}$ ähm ja die pflanzen zu unter̂stÜtzen;

$\rightarrow \quad 14 \quad$ wei für mich is_'WICHtig?

15 un un dass äh $^{\circ} \mathrm{h}$ dass die pflanzen KÖNnen mit den schädlingen 1Eben;

$16 \quad \mathrm{o}$ wir müssen eben nur`dAfür sorgen dass die pflanzen 'sO 
'STARK 'sInd dass: denen das nichts $\uparrow ` A u s m a c h t ;$

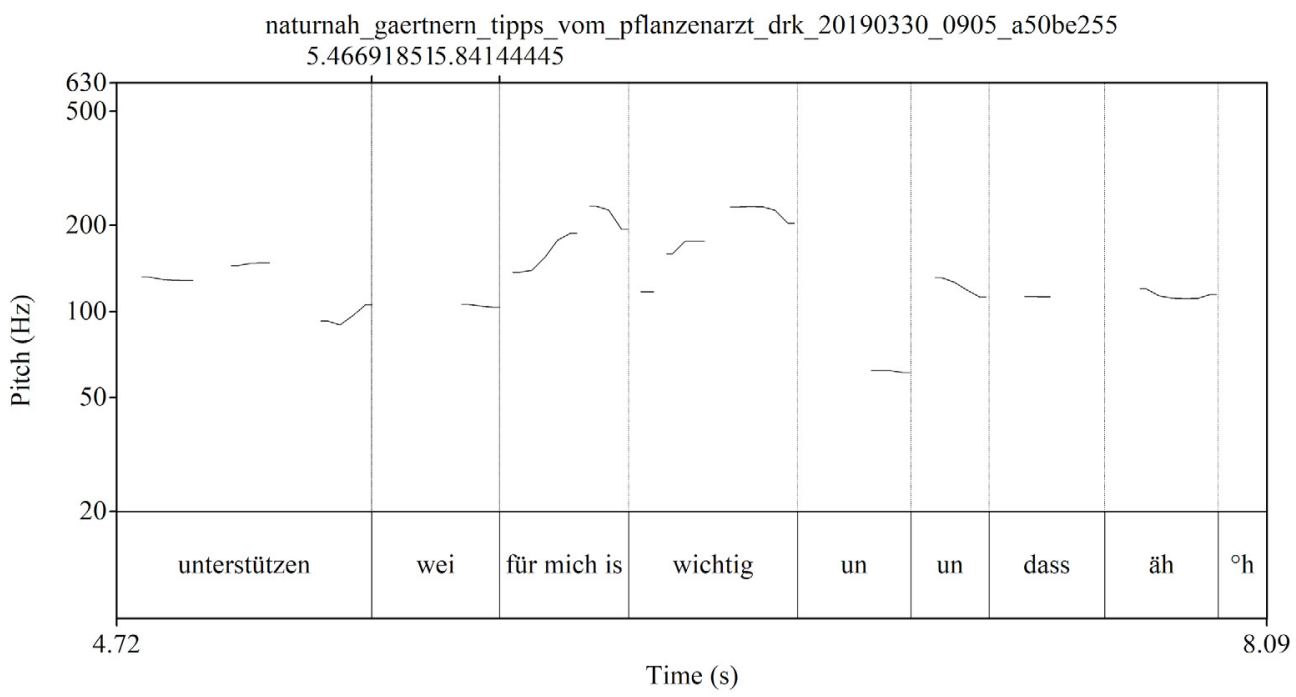

Abb. 1: Grafische Darstellung des Intonationsverlaufs einer weil-Äußerung, der keine Verzögerungssignale unmittelbar vorausgehen und die selbst keine Verzögerungssignale nach weil enthält

Bei diesem Typ von subjektiven (im Sinne von vom Sprecher ausgehenden) Meinungsäußerungen in Form von weil-Sätzen kann es sich ebenfalls um den Ausdruck eigener Gefühle handeln. So äußert der Sprecher in Zeile 25 des nächsten Transkripts ein Gefühl, das die eigentlich nicht mehr zu seinen aktuellen beruflichen Aufgaben passende Tätigkeit aus einer emotionalen Bindung zu seiner früheren Tätigkeit als Koch erklärt. Auf die Frage der Moderatorin der Sendung nach seiner aktuellen Stellung in seinem Betrieb, antwortet der Gast, dass er jetzt Manager und Koordinator ist, der aber das Kochen vermisst und deswegen jede Woche Gelegenheit zum Kochen sucht:

Transkript 5: ,Koch sein`

23 Ga: aber ich möchte immernoch koch SEIN;=

$24 \quad=$ und deswegen versuche ich immer ${ }^{\circ} \mathrm{hhh}$ ein zwei mal die woche doch noch in einem der restaurants zu 'KOchen, $(--)$

$\rightarrow \quad 25$ weil mir $\uparrow^{-}$FEHLT ,creaky>das>

Meinungs- bzw. gefühlsbezogene weil-Äußerungen mit Verbzweitstellung sind außerdem durch stärkere Tonhöhenakzente charakterisiert, als dies bei den weil-Äußerungen mit Verbletztstellung der Fall ist, wahrscheinlich weil sie eine stärkere emotionale Beteiligung voraussetzen. Im Gegensatz zu subjektiven Meinungsäußerungen und Gefühlsausdrücken in Form von weil-Sätzen mit Verbzweitstellung werden in der Mehrheit der weil-Sätze mit Verbletztstellung im hier benutzten Korpus Tatsachen benannt, die generellere Bekanntheit und allgemeinere Geltung beanspruchen können.

Im folgenden Transkript 6 befürwortet der Gast der Sendung konfrontative Gespräche, die ihm dazu verhalfen, anders Gesinnte zu begreifen. Dass er Meinungsverschiedenheit in Gesprächen gut verträgt, begründet er durch seine positive Einstellung in Bezug auf die Meinungsverschiedenheit überhaupt in Zeile 25, in welcher er einen weil-Satz mit Verbzweitstellung äußert.

Transkript 6: ,unterschiedliche Meinungen'

$20 \mathrm{Ga}$ ich bin sehr ge $\mathrm{A}^{\wedge} \mathrm{SPANNT}$ auf die ge ${ }^{\wedge}$ sprÄche;

21 wir sind gerade auf ${ }^{\wedge}$ TOUR mit der offenen gesellschaft an mehreren stati'Onen; 
23 Mo: $[\mathrm{mhm}]$

24 Ga: und wo ich mit anderen ${ }^{\wedge}$ MEInungen konfrontiert war und ich diesen austausch $\uparrow$ 'gUt fand; $m$ ?

weil ${ }^{\circ} \mathrm{h}$ s_is auch völlig in $\uparrow^{\prime}$ ORDnung dass man nicht einer meinung ist nach diesen ge^sprÄchen aber der $^{\wedge}$ Austausch ist extrem wichtig und;

\subsection{Weil-Äußerungen, die unmittelbar vor oder nach weil ein Verzögerungssignal zeigen}

Bei dieser Gruppe von weil-Äußerungen finden sich als hörbare Spuren von Denkprozessen Verzögerungssignale unmittelbar vor dem einleitenden weil oder Verzögerungssignale oder Pausen nach dieser Konjunktion. Dieser Tatbestand wird hier so interpretiert, dass es sich bei der folgenden Proposition um eine Begründung handelt, die erst ad hoc geplant und realisiert wird, nachdem die zu begründende Proposition, Folgerung oder eine ähnliche Sprechhandlung geäußert worden ist.

Im folgenden Gesprächsausschnitt ist die Rede von der Schädlichkeit erhöhter Aktivität vor dem Schlaf für die erste Schlafphase, für welche der tiefe Schlaf ideal ist. In Z. 27 wird daher davon abgeraten. Anschließend wird nach einem gedehnten weil und einem Verzögerungssignal ein Satz mit Verbzweitstellung geäußert. In ihm wird der allgemeine Hinweis zum Verhalten vor dem Schlafengehen aus Zeile 27 mit der möglichen Störung der ersten Schlafphase durch solche Aktivitäten begründet.

Transkript 7: , aufregende Dinge vor dem Schlaf ${ }^{\star}$

27 Ga: und ${ }^{-}$dEswegen ${ }^{\circ} \mathrm{h}$ sollte man auch drauf achten dass man eben vor dem zubettgehen ${ }^{\circ} \mathrm{h}$ ähm nicht zu viel aufregende dinge 'MACHT, wei:l äh sonst wird eben der schlaf am beginn ge'STÖRT.

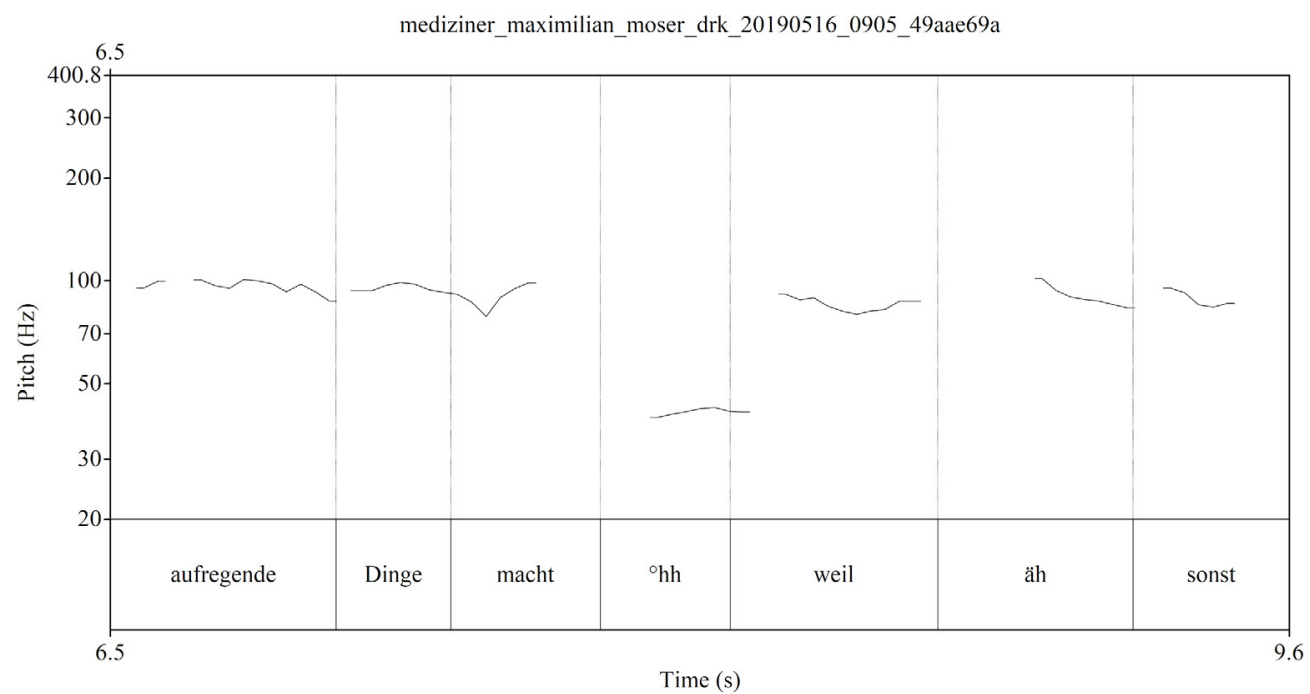

Abb. 2: Grafische Darstellung des Intonationsverlaufs einer weil-Äußerung, der ein hörbares Einatmen unmittelbar vorausgeht und die selbst ein Verzögerungssignal (äh) nach weil enthält

\section{4. weil als konversationelles Fortsetzungssignal?}

Das sogenannte konversationelle Fortsetzungssignal weil, dem keine Begründung oder Erklärung folgt, stellt m. E. eigentlich einen Teil der abgebrochenen weil-Äußerung dar, der anfänglich nicht 
als alleinstehendes Wort geplant war. Es erscheint nämlich immer an Stellen, vor welchen unmittelbar ein Begründungsbedarf entstanden ist.

Im folgenden Ausschnitt aus einem Gespräch über Fahrradfahrer im Verkehr folgt dem weil in Zeile 12 weder eine Begründung noch eine Erklärung o. Ä.

Transkript 8: ,der tote Winkel'

09 Ga: ,all `wenn sie eben rechts `ABbiegen; $=$

$10=$ und $^{\circ} \mathrm{hh}$ sich so langsam vortasten 'MÜSsen,

11 was sie auch defacto `MÜSsen um eben niemanden zu ge' fÄhrden,

$\rightarrow \quad 12$

13 ${ }^{\circ} \mathrm{h}$ weil ,p $>$ ich?〉 ,,all〉wir können die diskussion über den toten WINkel führen; $>=$ $=$ meiner meinung nach ${ }^{-}$GIBT es keinen toten 'wInkel,

Der Grund für das in den Zeilen 09 und 10 genannte langsame und vorsichtige Autofahren beim Rechtsabbiegen an der Kreuzung ist bereits in Zeile 11 in der durch um eingeleiteten Infinitivkonstruktion genannt worden: die Gefahr, jemanden zu überfahren, so dass danach kein Bedarf besteht, die Proposition aus den Zeilen 09 und 10 noch einmal zu begründen. Nach dem weil wird in den Zeilen 12 und 13 zunächst der tote Winkel thematisiert und dann die Meinung des Gastes der Sendung zum Ausdruck gebracht, dass bei hinreichend vorsichtigem Fahren kein toter Winkel auftrete. Dass diese zwei Propositionen, nämlich das langsame, vorsichtige Autofahren beim Rechtsabbiegen an der Kreuzung und der tote Winkel als Ausrede für unvorsichtiges Autofahren, zumindest nicht ohne eine kurze Einleitung der zweiten Proposition, aber jedenfalls nicht durch weil verbunden werden können, wird dem Gast unmittelbar nach geäußertem weil bewusst, wo die Konstruktion abgebrochen und eine neue angefangen wird.

\subsection{Verfestigte Verbzweitstellung in weil-Sätzen}

Im Folgenden werden Parallelen zwischen den Gruppen der weil-Äußerungen gezogen, die in den Unterkapiteln 3.1, 3.2 und 3.3 dieses Aufsatzes dargestellt sind. Für alle diese Gruppen gilt, dass die Verbindung der durch die weil-Äußerungen ausgedrückte Begründung bzw. Erklärung und der dazugehörigen Vorgängeräußerung nicht zum Wissen des Sprechers gehören und in der aktuellen Situation geplant und realisiert werden müssen. Der Begründungs- bzw. Erklärungsbedarf entsteht in diesem Fall erst nach der geäußerten Sprechhandlung, subjektiven Folgerung, Meinungsäußerung o. Ä.

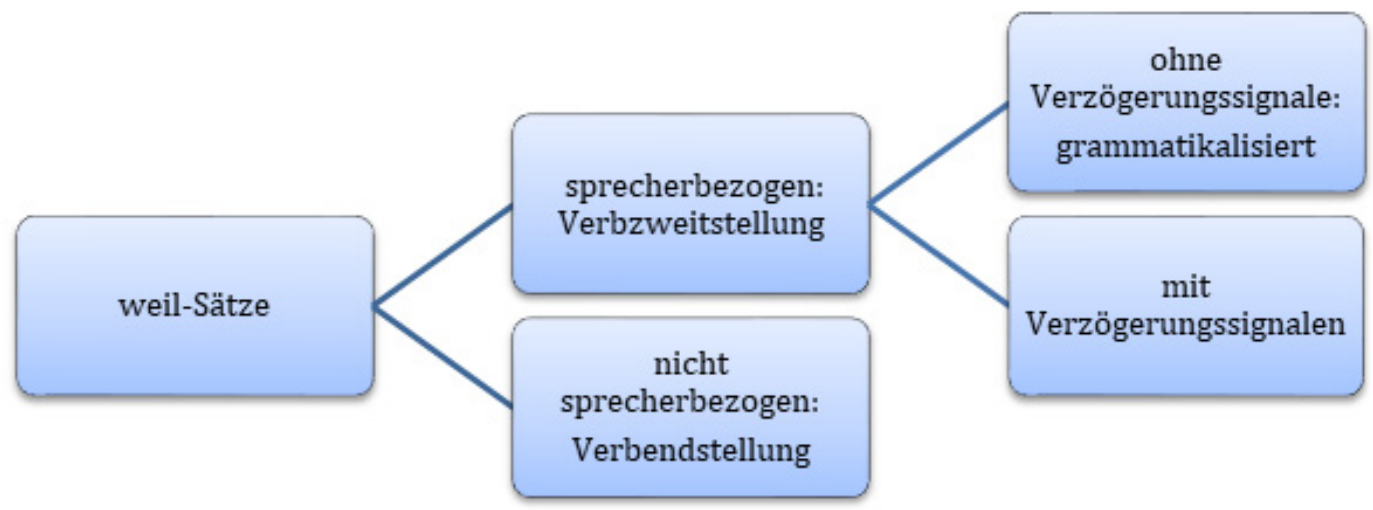

Abb. 3: Schematische Darstellung der weil-Äußerungen im gesprochenen Deutsch 
Verzögerungssignale unmittelbar vor der weil-Äußerung oder Pausen oder Verzögerungssignale in der weil-Äußerung direkt nach weil sind Symptome von Denkprozessen und stellen einen Abbruch des fließenden Sprechens dar und nach solch einer Unterbrechung erscheint die Wahl einer Hauptsatzverbstellung sinnvoll. Für die Gruppen der weil-Äußerungen ohne solche Verzögerungssignale oder Pausen wird angenommen, dass ihre Verbzweitstellung nicht durch einen späten Planungsmoment bedingt ist, sondern eine grammatikalisierte Option zum Ausdruck der Begründungen von Sprechhandlungen, subjektiven Folgerungen, Meinungs- und Gefühlsäußerungen sowie zum Ausdruck parenthetischer Zusatzinformationen darstellt. Allen diesen Begründungen bzw. Erklärungen ist ihre Sprecherbezogenheit gemeinsam, so dass von sprecherbezogenen weil-Äußerungen gesprochen werden könnte.

\section{Fazit}

Die Analyse von 100 weil-Äußerungen ergab, dass es sich bei allen weil-Äußerungen mit Verbzweitstellung um sprecherbezogene handelt, die zum Teil bereits aus der Fachliteratur bekannt sind (epistemische, sprechhandlungsbezogene Kausalrelation bzw. für das Verständnis wichtige Zusatzinformation) und zum anderen Teil meinungs- bzw. gefühlsbezogene Äußerungen darstellen, die in bisherigen Forschungen der weil-Äußerungen im gesprochenen Deutsch nicht erfasst worden sind. Die Verbzweitstellung der weil-Äußerungen im gesprochenen Deutsch weist außerdem darauf hin, dass diese weil-Äußerungen erst nach einem geäußerten Vorgängersatz ad hoc geplant werden, weil der Begründungs- bzw. Erklärungsbedarf im Grunde erst nach einem geäußerten Vorgängersatz entsteht. Die hinausgezögerten Denkprozesse, die bis nach weil reichen können, hinterlassen häufig eine Unterbrechung und daraus resultierende Verbzweitstellung, die üblicherweise neue Äußerungseinheiten kennzeichnet. Die Verbzweitstellung bei weil-Äußerungen, die ohne Hinauszögerung der Denkprozesse ad hoc geplant werden, ist bereits grammatikalisiert.

\section{Literaturverzeichnis}

FREYWALD, Ulrike (2009): Kontexte für nicht-kanonische Verbzweitstellung: V2 nach dass und Verwandtes. In: ReICH, Ingo / ReIs, Marga (Hrsg.): Koordination und Subordination im Deutschen. Hamburg, S. 113-134.

GÜNTHNER, Susanne (1993): “... weil - man kann es ja wissenschaftlich untersuchen“-Diskurspragmatische Aspekte der Wortstellung in WEIL-Sätzen. In: Linguistische Berichte 143, Hamburg, S. 37-59.

GüNTHNER, Susanne (1996): From Subordination to Coordination? Verb-second Position in German Causal and Concessive Constructions. In: Pragmatics 6, 3, Amsterdam, Philadelphia, S. 323-356.

GüNTHNER, Susanne und GoHL, Christine (1999): Grammatikalisierung von weil als Diskursmarker in der gesprochenen Sprache. In: Zeitschrift für Sprachwissenschaft, 18.1, Berlin, S. 39-75.

Scheutz, Hannes (1998): weil-Sätze im gesprochenen Deutsch. In: HuTTERER, Claus Jürgen / Pauritsch, Gertrude (Hrsg.): Beiträge zur Dialektologie des ostoberdeutschen Raumes. Referate der 6. Arbeitstagung für bayerisch-österreichische Dialektologie 1995 in Graz. Göppingen, S. $85-112$.

Scheutz, Hannes (2001): On causal clause combining. The case of weil in spoken German. In: Selting, Margret/Couper-Kunlen, Elizabeth(Hrsg.): Studies in Interactional Linguistics. Amsterdam, Philadelphia, S. 111-139.

SELTING, Margret et al. (2009): Gesprächsanalytisches Transkriptionssystem 2 (GAT 2). Gesprächsforschung - Online-Zeitschrift zur verbalen Interaktion, Bd. 10, S. 353-402, http://www. gespraechsforschung-ozs.de/ [23.11.2020.]. 